\title{
Performance Evaluation and Deployment Testing of Call Admission Control in IP Bearer Network
}

\author{
Lushanur Rahman, Molla Shahadat Hossain Lipu, Foisal Mahedi Hasan, Rabiul Islam Jony, and \\ Rejwanur Rashid Mojumdar
}

\begin{abstract}
The paper addresses the performance evaluation along with selection of descend percentage value selection, call barring alarm level and deploying test scenario of call admission control in an IP bearer network. The premise of call admission control is to provide the reliability of call over the IP network when congestion happens. The system module includes the MSC Server, Media Gateway, and Router. The software component includes the program and code implemented via manageable client interface. In its completion, the call admission control service was planned and designed in the manner that it functioned properly.
\end{abstract}

Index Terms-Internet Protocol Call Admission Control (IP CAC), Internet Protocol Quality of Service (IP QoS), Media Gateway (MGW), Mobile Switching Center (MSC) server, Call Barring Alarm Level (CBAL), Call Connection Ratio (CCR), First Decent Percentage (FDP), Second Decent Percentage (SDP).

\section{INTRODUCTION}

Compared with traditional TDM network, IP transport has a fatal weakness. It supposes that the bandwidth of IP backbone is large enough and always allows packet forwarding. It is not always true in reality, and no mechanism is defined on standard to reject the new call [1].

When IP backbone is congested, all ongoing service will be affected, IP QoS and IP CAC provide the reliability of call over the IP network [2]. For the career, in a mobile network, the IP QoS flow control ensures the quality of the IP bearer network [3]. In case of heavy traffic, the traffic can be efficiently distributed or subsequent calls are restricted so that the quality of established calls is maintained [4]. For the mobile subscribers the IP QoS flow control improves the quality of established calls.

When the QoS alarm generates and flow control function is activated, some call will be released according to the parameter configuration. In order to reduce the risk in existing IP bearer network, it is better to implement CAC in low traffic time. Call Admission can be controlled based on

Manuscript received October 19, 2012; revised November 25, 2012.

Lushanur Rahman is with the Department of Electronics and Department of communication Engineering, Tampere University of Technology (TUT), Tampere FI-33720, Finland (E-mail: lushan05@yahoo.com)

Molla Shahadat Hossain Lipu is with School of Environment, Resources and Development, Energy Field of Study, Asian Institute of Technology (AIT), Pathumthani 12120, Thailand (E-mail: lipuhossain@gmail.com)

Rabiul Islam Jony is with the Department of Communications and Networking, AALTO University, FI-00076 AALTO, Finland (Email: rabiuljony@yahoo.com)

Md. Rejwanur Rashid Mojumdar is with the Faculty of Engineering and Computer Science, University of Ulm, 89075 Ulm, Germany (E-mail: m.r.r.mojumdar@gmail.com) bandwidth or QoS or it simultaneously depends on both. If there is no available bandwidth or unacceptable QoS destination, CAC action will be launched in the network [5]. CAC action includes rejection of new call, reroute the call to TDM if available [2].

Organization of the rest of the paper is as follows: In section II and III, we discussed CAC enable Criterion and network scenario respectively. In section IV and V, we described the working principle of IP CAC in network and key parameters situation respectively. In section VI and VIII, we presented assumption and performance evaluation and testing. The paper ends with FDP and SDP value, call bearing alarm level control, conclusion and future work in section VIII, IX and IX respectively. This is our ongoing work here we simulated the result of packet Loss, call barring alarm level, call restriction percentage, call connection ratio, call success ration during CAC action, sub rotes overflow Items. In every case, the scheme is validated by simulation results. This paper mainly focuses on some important parameters such as CBAL, SDP and FDP required for implementation of IP CAC service. These parameters are based on real traffic scenario of a network operator.

\section{CAC ENABLE CRITERION AND ACTION}

Call attempt and termination between MSC servers are controlled by Bearer Independent Call Control (BICC) protocol. For bandwidth based CAC, MGW calculates the total bandwidth for the active call used. MGW compares the total bandwidth with the configured bandwidth [6]. If the total bandwidth is greater than the configured bandwidth, MGW will not assign the IP terminal for the call and the call will be rejected. Otherwise the call will be processed [7].

MSC Server monitors the sample call between different MGW. MGW calculates the IP QoS information according to the Real Time Controlling Protocol (RTCP). MGW periodically ( 5 seconds) reports the IP QoS information to the MSC Server through the monitoring sample call using the standard H.248 interface [8], [3]. MSC server will smoothly control the new incoming call using the QoS information. QoS detection on RTCP gives the information on time delay, jitter, and packet loss in the IP network. Reporting of Qos information related to IP bearer network to MSC server is done through Mc interface [3]. If overflow is detected, Flow Control initiates and improves the quality of established calls. Fig. 01 illustrates both bandwidth and QoS concept based action of CAC and its criterion in the mobile IP bearer network. 


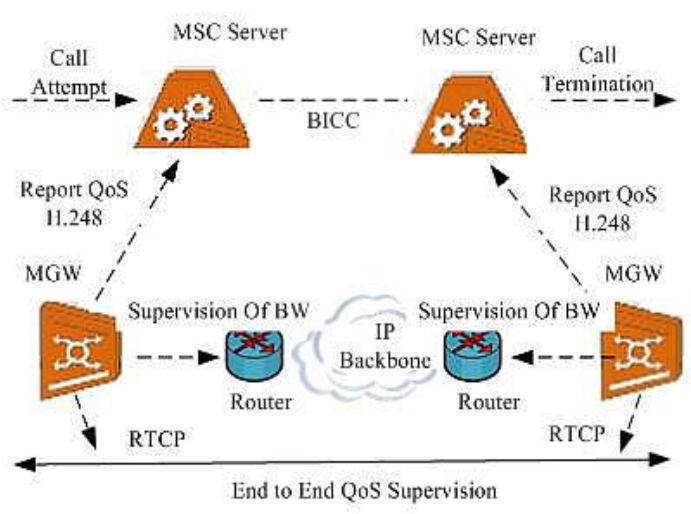

Fig. 1. CAC enabled criterion and action

\section{NETWORK SCENARIO}

The MSC which belongs to IP bearer network is classified into two network entities: MSC server and Media Gateway (MGW). One MSC server controls one or more MGWs through H.248 or Media Gateway Control Protocol (MGCP). The platform of MSC which we considered is Compact Peripheral component interconnect (CPCI), capacity of which is 450,000 , and registered subscribers are around 2 million. The resource utilization is around 50\% [8]. The functions of the network entities are as follows:

\section{A. Mobile Switching Center (MSC) server}

It is responsible for control functions in the circuitswitched (CS) domain, such as mobility management, security management, handoff processing, signaling processing, and subscriber service data management such as VLR function.

\section{B. Media Gateway (MGW)}

It bears the circuits in the CS network and media streams in the IP network, and is responsible for voice, data and video connection between the CS network and IP network, or in the same network.

\section{C. $\operatorname{Router}(R)$}

It is responsible for transporting voice traffic data between MGW and MGW. Several routers are used to make a native IP backbone transmission medium for carrying packet switched (PS) data [9].

The IP QoS flow control can be used when the MSC server works as end office, gateway office or tandem office. For an established call between two MGWs (intra-office or inter-office call), the IP QoS detection is implemented on the endpoints of the call. If an alarm is generated with the IP QoS damage, the MSC server restricts subsequent calls through the flow control mechanism such as quality degradation or call restriction, so that the voice quality of the established call is not affected [10].

\section{WORKING PRINCIPle OF IP CAC IN MSC SERVER}

In the IP QoS flow control, the functions of the MSC server and MGW are as follows:

- MGW reports the quality alarm for a call. The alarm value indicates the quality of the call. The greater the alarm value, the poorer the quality of the call.
- MSC server implements call sampling and the flow control of subsequent calls.

The sampling message sent by the MSC server to the gateway is used to detect the voice quality of calls in a certain direction on the gateway. If the voice quality is poor, subsequent calls are restricted, that is, the number of calls allowed per minute is restricted to a certain range. Through the sampling message, the QoS feedbacks of the bearer endpoints are given. Therefore, this method is also called feedback-mode flow control [4].

The implementation of the IP QoS flow control is as follows:

- The MSC server sends the call sampling message to the MGW before connecting to the calls in a certain direction on an MGW. After the connection, the MGW reports the alarm values of the voice quality of the calls to the MSC server.

- MSC server determines whether to implement the flow control for subsequent calls, based on the alarm value of the voice quality of the calls.

- The MSC server determines whether to implement the flow control for subsequent calls, based on the alarm value of the voice quality of the calls.

- When subsequent calls are connected, the flow control is implemented if the MSC server detects that the voice quality of the calls in a certain direction on the MGW becomes poorer. That is, the number of calls allowed per minute is restricted to a certain range. If the range is exceeded within a minute, the extra calls in this direction are released on the MGW. At the same time, a call restriction alarm is generated by the MSC server [9].

- When the voice quality of the calls of a certain direction becomes good, a recovery period is enabled to avoid the call traffic jitter during the recovery and a recovery alarm is generated.

-

\section{KeY PARAMETERS SitUATION}

IP CAC may influence call connection ratio (CCR) and overflow values. However, CCR and overflow values can be changed due to a lot of other factors; CCR and overflow values are treated as regular KPI for both TDM and IP call [10].

If IP CAC works at the time of congestion or poor QoS, then overflow will increase and CCR will decrease, and flow control is activated to ensure the voice quality of established calls, which means the new calls are restricted at the time of poor QoS [2]. The percentage of restricted calls along with the packet loss percentage along with QoS alarm level setting is shown in the following table.

\begin{tabular}{|} 
TABLE I: CALCULATED RESULT OF CALL RESTRICTION \\
\begin{tabular}{|c|c|c|}
\hline Packet Loss & Alarm level & Call Restriction \% \\
\hline$>=30 \%$ & 5 & $5 \%$ \\
\hline $15 \%-30 \%$ & 4 & $4 \%$ \\
\hline $8 \%-15 \%$ & 3 & $3 \%$ \\
\hline $3 \%-8 \%$ & 2 & $2 \%$ \\
\hline $0 \%-3 \%$ & 1 & $1 \%$ \\
\hline
\end{tabular}
\end{tabular}


The QoS alarm level has been defined at MGW end. MGW will inform about the poor QoS to the MSC server and then the server will start flow control.

\section{ASSUMPTIONS}

In deploying network, MSC has one MSC server and two MGW. QoS call control has one mode for outgoing calls, which is called Inter-office office direction mode [9].

The first assumption can be to enable the sampling function and IP QoS flow controls. The call control mode is Inter-office office direction mode because when the IP quality becomes low in one MGW, the subsequent calls are diverted to other MGWs with good bearer quality. If there are no other MGWs with good bearer quality, outgoing selection and subsequent calls fail [11].

To ensure minimum impacts possible it will be set to work only for the highest level (the higher the levels, the higher the packet loss occurs). For example, at level 5, 30\% packet loss will occur and it will restrict maximum 5\% outgoing calls towards any IP route. Without affecting the live call for test is not possible, we can just try to reduce the impact to live node .we set the "flow control" starting When the QoS alarm up to Level 5 and set the call descend percentage high value for example keeping up to $98 \%$ to reduce the impact to live service [11]. After detail calculation of traffic case of considered MSC we choose Call barring alarm level at 3 and FDP at 96\%, SDP at 95\% to improve the call quality during worse Qos and it will restrict call accordingly.

Overflow times along with sub-route is also observed. If flow control is activated in the route then voice quality will be improved after CAC action. Flow control has dependency with Minimum Threshold Time (MNT) value of call times in one minute. If the call times in one minute do not exceed this threshold value, the flow control will not be started. Now the value is set to 100 as per real traffic scenario. If MNT exceeds 100 then flow control will be initiated [9].

\section{Performance Evaluation And Deployment TESTING}

IP CAC test was performed in MSC, where it shuttled down the interface port intentionally from the router end to simulate the packet loss. Then Inter-Office office mode which worsened QoS alarm was generated automatically. For simplicity, the amount of signaling flows were ignored, because it was not significant compared to that of voice flows [10]. During the call test between two MSC's and after the transmission end port is down, call voice quality was found poor, and then the IP CAC flow control function was activated [12]. It was found through observation of Performance KPI, that some calls were rejected and the voice quality was improved as it was required.

\section{A. Test Methodology for IP CAC Deployment}

- Router end shut down the interface ports to simulate packet loss intentionally between two MGW's.
- Configure the IP CAC data, and set the "Call Barring Alarm Level (CBAL)" to level 3.

- Enable the flow control function in MSC server. In this scenario, the QoS alarm was generated and the flow control started. Adjust the FDP and SDP to $96 \%$ and $95 \%$.

All test procedure settings were done based on real traffic scenario and simulation.

\section{B. Performance KPI Analysis}

1) Sub Route Overflow times: For IP CAC, the all Subroute Over flow times were checked in Trunk office and the direction was of outgoing office traffic. From the performance analysis, data related to all IP sub routes are given in the table below.

TABLE II: SuB ROUTE Over Flow TIMES

\begin{tabular}{|c|c|c|c|}
\hline $\begin{array}{c}\text { Object } \\
\text { Instance }\end{array}$ & Start Time & $\begin{array}{c}\text { First Sub } \\
\text { routes } \\
\text { Overflow } \\
\text { Times }\end{array}$ & $\begin{array}{c}\text { Second Sub } \\
\text { routes } \\
\text { Overflow } \\
\text { Times }\end{array}$ \\
\hline IP Route 1 & $00: 20+06: 00$ & 22 & 10 \\
\hline IP Route 2 & $00: 50+06: 00$ & 34 & 6 \\
\hline IP Route 3 & $00: 40+06: 00$ & 16 & 0 \\
\hline IP Route 4 & $00: 30+06: 00$ & 13 & 3 \\
\hline
\end{tabular}

2) Overflow times: For IP CAC, the route over flows times were checked in Trunk office and the direction was of outgoing office traffic [12]. From the performance analysis, data related to all IP routes are given in the table below.

TABLE III: OVER FLOW TIMES IN OFFICE DIRECTION

\begin{tabular}{|c|c|c|c|}
\hline $\mathbf{3}$ & Start Time & $\begin{array}{c}\text { Call } \\
\text { Connected } \\
\text { Times }\end{array}$ & $\begin{array}{c}\text { Overflow } \\
\text { Times }\end{array}$ \\
\hline IP Route 1 & $00: 20+06: 00$ & 14 & 2 \\
\hline IP Route 2 & $00: 40+06: 00$ & 9 & 5 \\
\hline IP Route 3 & $00: 35+06: 00$ & 7 & 24 \\
\hline IP Route 4 & $00: 45+06: 00$ & 4 & 5 \\
\hline
\end{tabular}

\section{Call Connection Ratio}

After implementation of CAC to all the outgoing IP routes, the changes in call connected ratio along with other parameters are listed below.

\begin{tabular}{|c|c|c|c|c|}
\multicolumn{2}{c|}{ TABLE IV: CCR AFTER IP CAC IN IP ROUTE } \\
\begin{tabular}{|c|c|c|c|} 
Object \\
Instance
\end{tabular} & $\begin{array}{c}\text { Start } \\
\text { Time }\end{array}$ & $\begin{array}{c}\text { Connected } \\
\text { Ratio }\end{array}$ & $\begin{array}{c}\text { Answer } \\
\text { Ratio } \\
\text { Routes } \\
\text { overflow } \\
\text { Items }\end{array}$ & 66 \\
\hline IP Route 1 & $\begin{array}{c}03: 100 \\
+06: 00\end{array}$ & $96.43 \%$ & $25 \%$ & 79 \\
\hline IP Route 2 & $\begin{array}{c}03: 15 \\
+06: 00\end{array}$ & $85 \%$ & $25 \%$ & 1 \\
\hline IP Route 3 & $\begin{array}{c}03: 20 \\
+06: 00\end{array}$ & $98.89 \%$ & $15.56 \%$ & 10 \\
\hline IP Route 4 & $\begin{array}{c}03: 25 \\
+06: 00\end{array}$ & $90.7 \%$ & $18.52 \%$ & \\
\hline & Average & $\mathbf{9 2 . 7 6 \%}$ & & \\
\hline
\end{tabular}

Call connected ratio along with other parameters before 
implementation of CAC are listed below.

Finally it has been seen that there was change in the CCR after implementation of CAC.

TABle V: Calculated Result OF Connected Ratio, ANSwer Ratio, FIRST SUB ROTES OVERFLOW ITEMS

\begin{tabular}{|c|c|c|c|c|}
\hline $\begin{array}{c}\text { Object } \\
\text { Instance }\end{array}$ & $\begin{array}{c}\text { Start } \\
\text { Time }\end{array}$ & $\begin{array}{c}\text { Connected } \\
\text { Ratio } \\
\text { (average) }\end{array}$ & $\begin{array}{c}\text { Answer } \\
\text { Ratio } \\
\text { (average) }\end{array}$ & $\begin{array}{c}\text { First Sub } \\
\text { Routes } \\
\text { overflow Items } \\
\text { (average) }\end{array}$ \\
\hline $\begin{array}{c}\text { All IP } \\
\text { Routes }\end{array}$ & $\begin{array}{c}02: 00 \\
+06: 00\end{array}$ & $\mathbf{9 7 . 4 3}$ & 22.87 & 0 \\
\hline
\end{tabular}

\section{FDP AND SDP VALUE}

The first percentage and the second percentage were achieved according to the real traffic analysis, but it did not depend on the call attempt times. Suppose, if a part of the transmission was down from the beginning, the traffic would have become less. So the FDP can be set to $96 \%$ and SDP to $95 \%$. The FDP and SDP values became less, and the traffic decrease trend was more rapid as call restriction increases [12]. Fig. 2 shows traffic trend during testing at different decent percentage value.

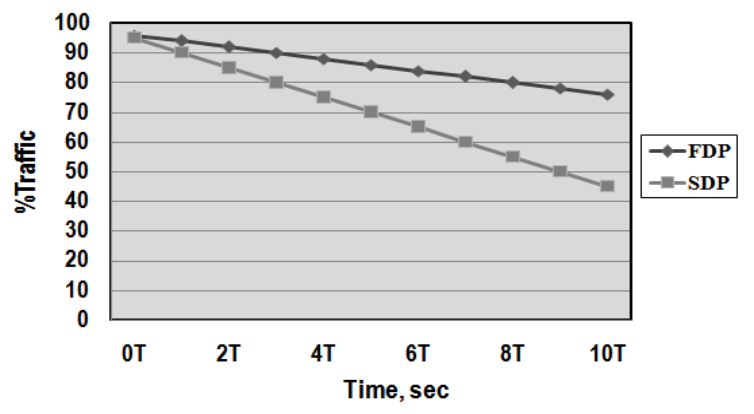

Fig. 2. FDP and SDP traffic scenario

\section{IMPROVEMENT Of CALl SuCCESS RATIO}

If Call Barring Alarm Level (CBAL) is set at three according to real traffic analysis by keeping FDP and SDP as it is, subscriber will experience better voice quality when IP CAC is in action, but without CAC this cannot be achieved. As a result, call success ratio of the established call increases during congestion or worse QoS period which is shown in the Fig. 3

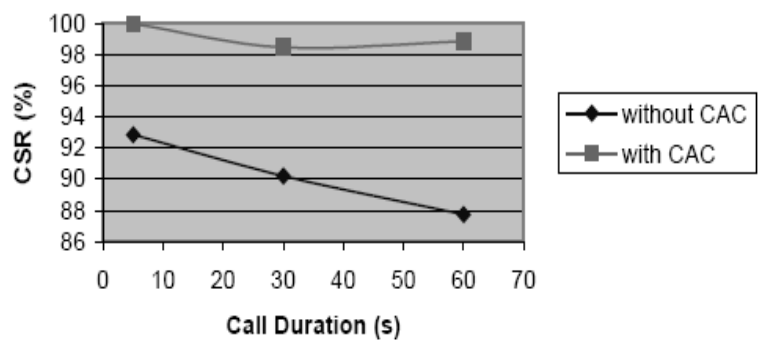

Fig. 3. CSR comparison with different call durations

\section{COnClusion And Future Work}

IP CAC can work properly, when there is packet loss for the IP network which can lead to bad voice quality. It can reject parts of calls to improve the voice quality and ensure better customer experience, which is a good opportunity for operators to improve the subscriber's satisfaction. The IP CAC flow control function can be enabled in live MSC according to the actual traffic mode. The only requirement to get benefits from CAC is to control the parameters of IP CAC. The admission thresholds need to be set properly for each end node pair. The relation of packet loss rate and the admission threshold is not obvious and it is not easy to find the optimal admission threshold analytically. Feedback control based on voice quality and packet loss measurements may be used to adjust the admission threshold dynamically. QoS is critical for the success of real time applications over IP, such as Voice over IP. On voice gateways, call admission control plays an important role for guaranteed QoS, for it makes decisions on whether and how to deliver the traffic based on different kinds of resources.

We show how system resources are explained on voice gateways help to guarantee the QoS of voice traffic. System resource module is shown for call admission control and traffic engineering. Network conditions are considered by several approaches to detecting connectivity and congestion. All the modules discussed here can be used as a part of CAC to guaranteed QoS, some procedures are recommended for integration in our paper.

As the rapid progress is being made in providing QoS, there are a lot of issues for better Call admission Control. For example, with RSVP enhancement for aggregation, CAC should be provided based on the aggregation policy instead of per call requirement. How to integrate some of the above CAC modules with DiffServ and IP/MPLS is another interesting topic

\section{ACKNOWLEDGEMENT}

At first we want to thank graceful ALMIGHTY for His blessings on us along the way. It is indeed a great privilege for us to express our heartiest gratitude and thankful appreciation to the organization, research groups, technical support teams, tools providers for supporting us in formulating our paper. All the necessary information, important graphs and diagrams, data provided by the organization have made it possible to complete this work successfully. We also want to take this opportunity to thank our family as we don't think we thank them enough for all they do for us. It could have been simply impossible to complete this work without their patience, love and constant encouragement.

\section{REFERENCE}

[1] V. E. Lek, G. Karlsson, and R. Ronngren, "Admission control based on end-to-end measurement," INFOCOM, 2000, 2000

[2] M. Naghshineh and M. Schwartz, "Distributed Call Admission Control in Mobile/Wireless Networks," IEEE J. Selected Areas in Comm., vol. 14, no. 4, pp. 711-717, May 1996.

[3] Quality of Service. [Online]. Available: http://www.cisco.com/warp/public/732/Tech/qos/

[4] D. Levine, I. Akyildiz, and M. Naghshineh, "A Resource Estimation and Call Admission Algorithm for Wireless Multimedia Networks Using the Shadow Cluster Concept," IEEE/ACM Trans. Networking, vol. 5, no. 1, pp. 1-12, Feb. 1997.

[5] S. Wu, K. Y. M. Wong, and B. Li, "A Dynamic Call Admission Policy with Precision QoS Guarantee Using Stochastic Control for 
Mobile Wireless Networks," IEEE/ACM Trans. Networking, vol. 10, no. 2, pp. 257-271, Apr. 2002.

[6] G. Bianchi, A. Capone, AND C. Petrioli, "Throughput analysis of end-to-end measurement-based admission control in IP," INFOCOM, 2000.

[7] B. Li, L. Yin, K. Y. M. Wong, and S. Wu, "An Efficient and Adaptive Bandwidth Allocation Scheme for Mobile Wireless Networks Using an On-Line Local Estimation Technique," ACM/Kluwer Wireless Networks, vol. 7, no. 2, pp. 107-116, 2001.

[8] L. Breslau, E. Q. Knightly, S. Shenker, I. Stoica, and H. Zhang, "Endpoint admission control: architectural issues and performance," pp. 57-69, SIGCOMM'00, 2000.

[9] B. M. Epstein and M. Schwartz, "Predictive QoS-Based Admission Control for Multiclass Traffic in Cellular Wireless Networks," IEEE J. Selected Areas in Comm., vol. 18, no. 3, pp. 523-534, Mar. 2000.

[10] A. Aljadhai and T. F. Znati, "Predictive Mobility Support for QoS Provisioning in Mobile Wireless Networks," IEEE J. Selected Areas in Comm., vol. 19, no. 10, pp. 1915-1930, Oct. 2001.

[11] S. Wu, K. Y. M. Wong, and B. Li, "A Dynamic Call Admission Policy with Precision QoS Guarantee Using Stochastic Control for Mobile Wireless Networks," IEEE/ACM Trans. Networking, vol. 10, no. 2, pp. 257-271, Apr. 2002.

[12] Y. Wang, "CAC Performance with Self-Similar Traffic," M.Sc. thesis, Department of Computer Science, University of Saskatchewan, Canada, August 1999.

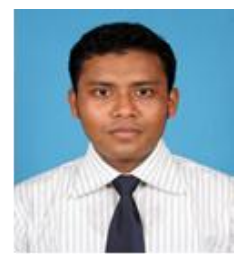

Lushanur Rahman was born in Dhaka, Bangladesh, 1987. He received his B.Sc. degree in Electrical and Electronic Engineering from Islamic University of Technology (IUT), Dhaka, Bangladesh in 2008. Currently he is pursuing his M.Sc. Degree in Electrical Engineering from Tampere University of Technology (TUT), Tampere, Finland.

He worked as an IP network Engineer in Robi Axiata Limited (One of the largest telecom operator in Bangladesh) from 2009 to 2011.He had the hands on experience with mobile backbone implementation and IP network routing. Currently he is engaged with The department of communication engineering of Tampere University of Technology, Finland. His current research interests are in the areas of wireless communications, Radio Frequency Engineering, communication circuits and systems, RFASIC design. Moreover he had contributed to several international conference papers on IP based communication in mobile telecommunication.

Mr. Rahman is an active student member of student union at TUT, Finland.

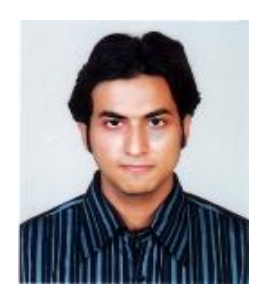

Molla Shahadat Hossain Lipu was born in Dhaka, Bangladesh, 1986. He completed his B.Sc. degree in Electrical and Electronic Engineering from Islamic University of Technology (IUT), Dhaka, Bangladesh in 2008. At present, he is pursuing his master degree in Energy field of study at Asian Institute of Technology (AIT).

He served as a lecturer in the Dept. of Electrical and Electronic Engineering at University of Asia Pacific from October, 2009 to July, 2011. Currently he is on study leave. Before this job he worked as a Lecturer in the Department of Mechatronics Engineering at World University of Bangladesh from February, 2009 to September, 2009. Mr. Molla Shahadat Hossain Lipu is an active student member of IACSIT. His current research interests are in the areas of renewable energy, energy planning and energy access. Moreover, he had contributed to several international conference papers.

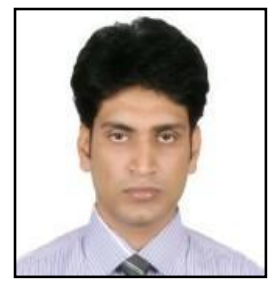

Foisal Mahedi Hasan was born in Dhaka, Bangladesh, 1986. He earned his B.Sc degree in electrical and electronic engineering from Islamic University of Technology, Dhaka, Bangladesh in 2008. Currently he is pursuing his M.Sc eegree in electrical engineering from The Petroleum Institute , Abudhabi, UAE

$\mathrm{He}$ worked as a core network planning engineer in Axiata Bangladesh Ltd (One of the largest telecom operator in Bangladesh) from 2009 to 2010. Currently he is working as research assistant in electrical engineering department of The Petroleum Institute, Abudhabi, UAE.

Md Hasan is an active student member of IEEE. His research interest focus on power quality, active filter, electric drives, multilevel converters as well as DSP based power electronics control.

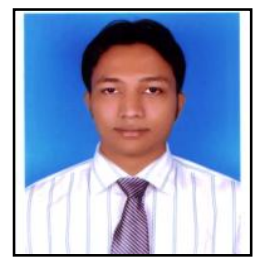

Rabiul Islam Jony was born in Dhaka, Bangladesh, 1986. He earned his B.Sc degree in Electrical and Electronic Engineering from Islamic University of Technology, Dhaka, Bangladesh in 2008. Currently he is pursuing his M.Sc eegree in Communications Engineering-Radio Communications from department of University, Finland. communications and networking of Aalto

He worked as a RF Engineer in Banglalion Communications Ltd (One of the largest WiMAX operator in Bangladesh) from Feb, 2011 to September, 2011. Before this job he worked as a Lecturer in Department of Mechatronics Engineering in World University of Bangladesh (One of top ranked private university in Bangladesh) from June, 2009 to January, 2011. Mr. Jony is an active student member of IACSIT. His research interests focus on Radio network planning and optimization, RF propagation models, Future mobile communications technology, LTE-A, Machine to Machine Communications.

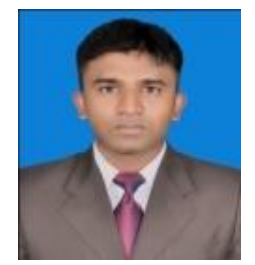

Rejwanur Rashid Mojumdar was born in Dhaka, Bangladesh at 20 August, 1986. He received his B.Sc. in electrical and electronic engineering from the Islamic University of Technology (IUT), (a subsidiary organ of the OIC), Bangladesh in November 2008. Currently, he has been studying in an international master's on energy science and technology at Ulm University, Germany. From September 2012, he is also enrolled to start a joint master's within European Institute of Innovation and Technology (EIT) on smart electrical networks and systems at the Royal Institute of Technology (KTH), Sweden.

Since April 2009 to April 2011, he had been working as a Site Engineer (SCADA) at NLDC (National Load Dispatch Centre) Project of AREVA T\&D Bangladesh. It was a Supervisory Control and Data Acquisition (SCADA) deployment project to bring the national power transmission substations (230/132/33 KV) under a central SCADA system and build the NLDC. Necessarily, he had visited almost all of the transmission substations and power stations in Bangladesh and skillfully served some imperative duties. From April 2011 to October 2011, he had been working as a Lead Energy Auditor of Deutsche Gesellschaft für Internationale Zusammenarbeit (GIZ) GmbH in ESIP (Energy Saving Initiative Project) on the Program of PSES (Promotion of Social and Environmental Standards in the Industry). He had led an ingenious team of energy auditors in the preparation of two investment grade energy audit reports as executive summary from this renowned federal enterprise. Moreover he had contributed to seven international journal papers on energy systems and sustainable development related topics and two international papers on communication technologies and one international conference paper on optics. Currently, he has deep research interest on grid integration of renewable energies, control systems, and various smart grid technologies. 